\title{
Estimation of Contact Forces using a Virtual Force Sensor
}

\author{
Emanuele Magrini Fabrizio Flacco Alessandro De Luca
}

\begin{abstract}
Physical human-robot collaboration is characterized by a suitable exchange of contact forces between human and robot, which can occur in general at any point along the robot structure. If the contact location and the exchanged forces were known in real time, a safe and controlled collaboration could be established. We present a novel approach that allows localizing the contact between a robot and human parts with a depth camera, while determining in parallel the joint torques generated by the physical interaction using the so-called residual method. The combination of such exteroceptive sensing and model-based techniques is sufficient, under suitable conditions, for a reliable estimation of the actual exchanged force at the contact, realizing thus a virtual force sensor. Multiple contacts can be handled as well. We validate quantitatively the proposed estimation method with a number of static experiments on a KUKA LWR. An illustration of the use of estimated contact forces in the realization of collaborative behaviors is given, reporting preliminary experiments on a generalized admittance control scheme at the contact point.
\end{abstract}

\section{INTRODUCTION}

Robotics research looks forward to the possibility of bringing closer humans and robots, when this is desired. Robots that can physically collaborate with humans will combine and enhance the skills of both the robot and the human. This will have multiple potential applications in industrial robotics (robot co-workers) and service robotics (personal assistants). To this end, robots have to be designed and controlled following new guidelines. We have recently proposed in [1] a control framework for safe physical HumanRobot Collaboration (pHRC), based on a stack of nested consistent behaviors of the robot, see Fig. 1. Safety is the most important low-level feature of a robot that should work with humans, which includes the ability of fast detection and reaction to collisions. Coexistence is the robot capability of sharing its workspace, without interfering with humans. Collaboration occurs when the robot performs a complex task with direct human interaction and coordination.
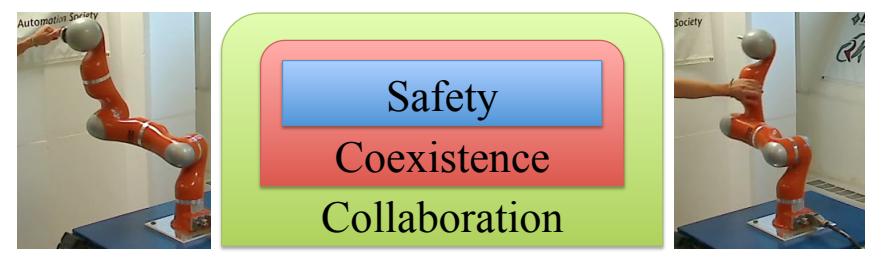

Fig. 1. Stack of nested consistent behaviors for pHRC [1]

The authors are with the Dipartimento di Ingegneria Informatica, Automatica e Gestionale, Sapienza Università di Roma, Via Ariosto 25, 00185 Roma, Italy (\{magrini,fflacco,deluca $\} @$ diag.uniroma1.it). This work is supported by the European Commission, within the FP7 ICT-287513 SAPHARI project (www.saphari.eu).
We concentrate here on the physical human-robot collaboration aspects, in which a human enters in contact with a robot and the robot reacts as a function of the exchanged forces. According to our nested paradigm, this implies that safe coexistence is already possible and continuously guaranteed. In order to physically collaborate, the robot has to accomplish four subtasks:

1) detect a contact with the human, and distinguish between intentional contact or undesired collision;

2) identify the point on the robot surface where the contact has occurred;

3) estimate the exchanged Cartesian forces;

4) control the robot to react according to a desired behavior.

Different model-driven and/or sensor-driven methods can be used to detect a contact (or collision). The collision detection system of [2] relies on a nonlinear adaptive impedance control law, while an image-based collision detector was used in [3]. An efficient residual-based approach has been presented in [4], [5]. In [6], an on-line filtering action on the motor currents allows discriminating between desired contacts and collisions in an industrial robot.

Once a contact has been detected, it is still not immediate to identify the contact location on the robot surface. A way to address this problem is to resort to a tactile sensor skin distributed along the whole surface of the robot, e.g., as in [7] where also the direction and intensity of the force is being measured. A less invasive approach is to use vision systems [8]. In [9], data from a 6D wrist F/T sensor are combined with exploratory robot motions, and a probabilistic approach is followed to localize the contact. A method to estimate constant Cartesian forces acting during contact without the use of torque sensing and with the robot at rest, is presented in [10].

Once the contact point is known and the exchanged forces are measured (or estimated), there are a number of different control laws in the literature aimed at regulating the contact behavior. The most common is probably impedance control [11], in which a desired behavior is assigned to the end-effector contact based on a mass-spring-damper model. In particular, in order to change the apparent mass/inertia with impedance control, knowledge of the contact force is needed. Special impedance (or compliance) controllers have been developed also for robots with flexible joints [12] [14] and for redundant robots [15]. Another possibility is to implement admittance control [16], where robot velocity is commanded in response to an applied force.

In this paper, we focus mainly on the collaboration subtasks 1) to 3) listed above. Combining the geometric information about the contact, as localized online by a depth 
sensor, with the evaluation of the joint torques resulting from a contact, as obtained by our standard residual-based method, allows estimating the exchanged Cartesian forces at any contact point along the robot structure, without the need of force or torque sensing. Under suitable hypotheses, such a virtual sensor procedure can handle also multiple point contacts and/or the inclusion of contact torques. The estimate of contact forces can be used then in the design of admittance, impedance, or force control laws. In all cases, the reference behavior is conveniently specified and controlled at the contact, rather than at the joint or end-effector level.

The paper is organized as follows. Section II recalls the basics used in the paper. Section III details how information on Cartesian contact forces can be extracted from the residualbased estimation of the external joint torques. On-line detection of the contact point using a depth camera is presented in Sect. IV. Finally, Section V reports several experimental results with the proposed contact force estimation method and its use for controlling physical interaction between a human and a KUKA LWR robot.

\section{PRELIMINARIES}

We consider a robot manipulator as an open kinematic chain of $n+1$ rigid links, connected by $n$ joints and with generalized coordinates $\boldsymbol{q} \in \mathbb{R}^{n}$. The position and orientation of a frame attached to link $i$ with respect to the (base) frame 0 is expressed by the homogeneous matrix

$$
\boldsymbol{T}_{i}(\boldsymbol{q})=\left(\begin{array}{cc}
\boldsymbol{R}_{i}(\boldsymbol{q}) & \boldsymbol{p}_{i}(\boldsymbol{q}) \\
\mathbf{0}^{T} & 1
\end{array}\right), \quad i=1, \ldots, n,
$$

where $\boldsymbol{R}_{i}$ is the rotation matrix of frame $i$ and $\boldsymbol{p}_{i}$ is the position of the origin of frame $i$. The $(6 \times n)$ geometric Jacobian associated to link $i$ relates the joint velocity $\dot{\boldsymbol{q}}$ to the linear velocity $\boldsymbol{v}_{i} \in \mathbb{R}^{3}$ of the origin of frame $i$ and to the angular velocity $\boldsymbol{\omega}_{i} \in \mathbb{R}^{3}$ of frame (viz. link) $i$ as

$$
\left(\begin{array}{c}
\boldsymbol{v}_{i} \\
\boldsymbol{\omega}_{i}
\end{array}\right)=\boldsymbol{J}_{i}(\boldsymbol{q}) \dot{\boldsymbol{q}}
$$

where the last $n-i$ columns of $\boldsymbol{J}_{i}$ are identically zero.

With reference to Fig. 2, consider a generic (contact) point on the surface of the $i$-th link, whose absolute position is

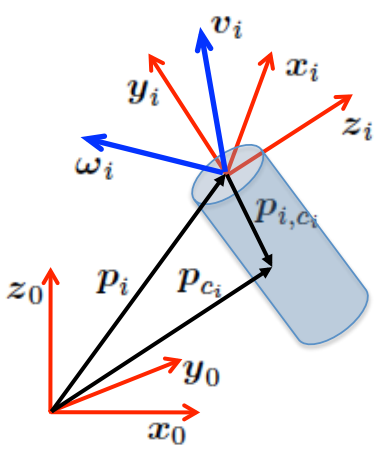

Fig. 2. The generic link $i$ of a robot, with its kinematic quantities and the location of a contact point given by $\boldsymbol{p}_{c_{i}}$. Its position with respect to the origin of frame $i$ is given by

$$
\boldsymbol{p}_{i, c_{i}}(\boldsymbol{q})=\boldsymbol{p}_{c_{i}}(\boldsymbol{q})-\boldsymbol{p}_{i}(\boldsymbol{q})
$$

We shall need the $(6 \times n)$ geometric Jacobian matrix $\boldsymbol{J}_{c}$ associated to a contact situation on link $i$ (we dropped for compactness the index $i$ of the link under consideration). This is obtained from (1) and (2) as

$$
\boldsymbol{J}_{c}(\boldsymbol{q})=\left(\begin{array}{c}
\boldsymbol{J}_{L, c}(\boldsymbol{q}) \\
\boldsymbol{J}_{A, c}(\boldsymbol{q})
\end{array}\right)=\left(\begin{array}{cc}
\boldsymbol{I} & -\boldsymbol{S}\left(\boldsymbol{p}_{i, c_{i}}(\boldsymbol{q})\right) \\
\boldsymbol{O} & \boldsymbol{I}
\end{array}\right) \boldsymbol{J}_{i}(\boldsymbol{q}),
$$

where $\boldsymbol{S}(\boldsymbol{v})$ denotes the skew-symmetric matrix built with the components of vector $\boldsymbol{v}$. The two $(3 \times n)$ matrices $\boldsymbol{J}_{L, c}$ and $\boldsymbol{J}_{A, c}$ in the partition of $\boldsymbol{J}_{c}$ relate the joint velocity $\dot{\boldsymbol{q}}$, respectively to the linear velocity $\boldsymbol{v}_{c}=\dot{\boldsymbol{p}}_{c}$ of the contact point on link $i$ and to the angular velocity $\boldsymbol{\omega}_{i}$ of link $i$. The Jacobian matrix $\boldsymbol{J}_{c}$ has the last $n-i$ columns identically zero, when it refers to a contact occurring on link $i$.

Suppose that an external force $\boldsymbol{F}_{c} \in \mathbb{R}^{3}$ is applied to the contact point $\boldsymbol{p}_{c}$ on link $i$, and that an external torque $M_{c} \in \mathbb{R}^{3}$ is applied to the same link. These two vectors can be arranged in an external generalized force $\Gamma_{c} \in \mathbb{R}^{6}$ that produces a joint torque (performing work on $\boldsymbol{q}$ ), which is expressed, by duality from (3), as

$$
\boldsymbol{\tau}_{e x t}=\boldsymbol{J}_{c}^{T}(\boldsymbol{q}) \boldsymbol{\Gamma}_{c}=\left(\begin{array}{ll}
\boldsymbol{J}_{L, c}^{T}(\boldsymbol{q}) & \boldsymbol{J}_{A, c}^{T}(\boldsymbol{q})
\end{array}\right)\left(\begin{array}{c}
\boldsymbol{F}_{c} \\
\boldsymbol{M}_{c}
\end{array}\right)
$$

Note that, in general, both $\boldsymbol{\Gamma}_{c}$ and the contact point $\boldsymbol{p}_{c}$ (and thus, the associated Jacobian $\boldsymbol{J}_{c}$ ) are unknown in the absence of other external/tactile sensors.

Finally, the dynamic model of a rigid robot is

$$
\boldsymbol{M}(\boldsymbol{q}) \ddot{\boldsymbol{q}}+\boldsymbol{C}(\boldsymbol{q}, \dot{\boldsymbol{q}}) \dot{\boldsymbol{q}}+\boldsymbol{g}(\boldsymbol{q})=\boldsymbol{\tau}+\boldsymbol{\tau}_{e x t},
$$

where $M$ is the symmetric, positive definite inertia matrix, $\boldsymbol{C} \dot{\boldsymbol{q}}$ includes the Coriolis and centrifugal terms, $\boldsymbol{g}$ is the gravity term, $\tau$ is the control torque, and $\tau_{\text {ext }}$ is the torque in (4) due to external/environmental generalized contact forces acting on the robot.

\section{Contact Force Estimation}

Physical collaboration is characterized by an exchange of contact forces/torques between the human and the robot, which may occur at any place along the robot structure. Detection of contacts is a fundamental feature for safe pHRI and must be very efficient, in order to allow fast and reliable robot reaction. For this purpose, we start from the residualbased approach of [5], which allows both dynamic contact detection and isolation of the link involved. This method is based on the dynamic model (5), and requires only the joint position measures provided by the encoders and the applied control torque. Moreover, in the presence of elasticity at the robot joints, the same detection scheme can be implemented very easily by using joint torque sensing, see [17]. 


\section{A. External Joint Torque Estimation}

The residual vector $\boldsymbol{r} \in \mathbb{R}^{n}$ is defined as

$\boldsymbol{r}(t)=\boldsymbol{K}_{I}\left(\boldsymbol{p}-\int_{0}^{t}\left(\boldsymbol{\tau}+\boldsymbol{C}^{T}(\boldsymbol{q}, \dot{\boldsymbol{q}}) \dot{\boldsymbol{q}}-\boldsymbol{g}(\boldsymbol{q})+\boldsymbol{r}\right) d s\right)$,

where $\boldsymbol{p}=\boldsymbol{M}(\boldsymbol{q}) \dot{\boldsymbol{q}}$ is the generalized momentum of the robot and $\boldsymbol{K}_{I}>0$ is a diagonal gain matrix. The residual can be directly obtained from the FastResearchInterface in the controller of the KUKA LWR robot (even if suitable digital filtering should be applied to this output). However, we note also that $\boldsymbol{r}$ can be computed for any other robot, starting from its dynamic model and using (6). The dynamic evolution of $\boldsymbol{r}$ has the stable, first-order filter structure

$$
\dot{\boldsymbol{r}}=\boldsymbol{K}_{I}\left(\boldsymbol{\tau}_{e x t}-\boldsymbol{r}\right),
$$

so that we can assume, for sufficiently large gains,

$$
\boldsymbol{r} \simeq \boldsymbol{\tau}_{\text {ext }} .
$$

This approach provides a model-based estimate of the external joint torque resulting from a contact force/torque applied anywhere to the robot. Based on (7), contact is detected when the residual exceeds some suitable threshold, e.g., when $\|\boldsymbol{r}\|>r_{t}$ with $r_{t}>0$, so as to prevent false alarms due to measurement noise and/or model uncertainties in (6).

The force estimation procedure that follows can be activated for any type of physical contact, but it is more useful when a soft contact is recognized by using the filtering method in [6]. In our approach, we have classified as collision any hard and fast contact that is able to excite a highpass filtered version of the estimated external joint torque. Otherwise, the contact is classified as soft and signalizes a request of physical collaboration by the human, rather than the occurrence of an undesired collision.

\section{B. Dealing with Contact Forces}

We will assume from now on that only point-wise forces $\boldsymbol{F}_{c}$ are applied by the human to the robot structure. While the process can be devised in principle also for estimating a complete six-dimensional generalized force/torque vector $\boldsymbol{\Gamma}_{c}$, the motivation for this simplifying assumption is twofold:

i) most intentional contacts with a single hand or arm, or with the human fingers, are not able to transfer a nonnegligible torque $\boldsymbol{M}_{c}$ to the robot, and this is especially true for fast or impulsive-type contacts;

ii) in order to estimate properly $\boldsymbol{\Gamma}_{c}$, i.e., both a contact force $\boldsymbol{F}_{c}$ applied at a point of a link and a contact torque $\boldsymbol{M}_{c}$ acting on the link, we should have rank $\boldsymbol{J}_{c}=6$, which is true only if the robot has $n \geq 6$ joints and the involved link is either the last one (when $n=6$ ) or sufficiently close to the end-effector (for $n>6$ ).

This second motivation is particularly relevant, since we would like to consider contacts along the entire chain structure of the robot, i.e., also for a link $i$ with $i<6$. We shall see in Sect. V that restricting the estimation only to contact forces $\boldsymbol{F}_{c}$ leads to more robust numerical results, e.g., even when the human touches the robot using the whole hand.
Therefore, setting $\boldsymbol{M}_{c}=\mathbf{0}$ in (4), we shall consider only cases when

$$
\boldsymbol{\tau}_{e x t}=\boldsymbol{J}_{L, c}^{T}(\boldsymbol{q}) \boldsymbol{F}_{c} .
$$

The dimension of the task related to the contact force is thus $m=3$. Combining eqs. (7) and (8), and dropping the index $L$ from the Jacobian to simplify notation, we need to solve, at the current configuration $\boldsymbol{q}$, the system of $n$ linear equations

$$
\boldsymbol{r}=\boldsymbol{J}_{c}^{T}(\boldsymbol{q}) \boldsymbol{F}_{c}
$$

for the unknown vector $\boldsymbol{F}_{c} \in \mathbb{R}^{3}$. Depending on which link in the kinematic chain is involved in the contact, the last few components of the residual vector $r$ evaluated by (6) can be zero (up to noise), while the associated rows of the transpose Jacobian $\boldsymbol{J}_{c}$ are structurally zero as well. Accordingly, equation (9) may consist of a square, under-, or over-determined linear system. The external contact force will always be estimated by pseudoinversion ${ }^{1}$ as

$$
\widehat{\boldsymbol{F}}_{c}=\left(\boldsymbol{J}_{c}^{T}(\boldsymbol{q})\right)^{\#} \boldsymbol{r}
$$

It should be stressed that the estimate $\widehat{\boldsymbol{F}}_{c}$ will be limited only to those components of $\boldsymbol{F}_{c}$ that can be detected by the residual $\boldsymbol{r}$. In fact, all forces $\boldsymbol{F}_{c} \in \mathcal{N}\left(\boldsymbol{J}_{c}^{T}(\boldsymbol{q})\right)$ will never be recovered in $\widehat{\boldsymbol{F}}_{c}$. This should not be considered a serious limitation, because such force components do not produce active work, and are absorbed by the robot structure (they are not dangerous for the human safety).

Putting together (9) and (10), the estimated Cartesian force can be expressed as

$$
\widehat{\boldsymbol{F}}_{c}=\left(\boldsymbol{J}_{c}^{T}(\boldsymbol{q})\right)^{\#} \boldsymbol{J}_{c}^{T}(\boldsymbol{q}) \boldsymbol{F}_{c} .
$$

We should consider the case when the contact Jacobian is not full rank. This situation is encountered when contact occurs at a link close to the robot base (the number of useful residuals is smaller than $m=3$ ) or when the manipulator (or a sub-chain of it) is in a singular configuration. Although these two cases may seem different, the mathematical result is the same, namely that $\left(\boldsymbol{J}_{c}^{T}\right)^{\#} \boldsymbol{J}_{c}^{T} \neq \boldsymbol{I}$, so that part of the contact forces may not be identified.

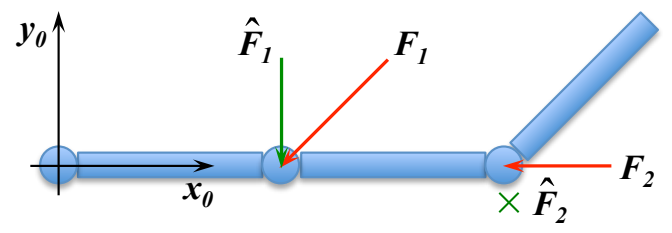

Fig. 3. Illustrative example of problems in contact force estimation

To illustrate this basic problem, consider a planar $3 \mathrm{R}$ manipulator with links of unitary length in the configuration $(0,0, \pi / 4)$, as shown in Fig. 3. In this case the task space of contact forces is reduced to $m=2$. When the contact occurs

\footnotetext{
${ }^{1}$ When the contact Jacobian is close to a singularity, numerical robustness is obtained by using a damped least squares method.
} 
on link 1, the actual contact force cannot be estimated (except for forces lying entirely in the $\boldsymbol{y}_{0}$-direction). For example, the force $\boldsymbol{F}_{1}=(-1-1)^{T}$ is estimated as $\hat{\boldsymbol{F}}_{1}=(0-1)^{T}$. On the other hand, the force $\boldsymbol{F}_{2}=\left(\begin{array}{ll}-1 & 0\end{array}\right)^{T}$ acting on the second link is completely absorbed by the structure, and so $\hat{\boldsymbol{F}}_{2}=\mathbf{0}$ (because $\boldsymbol{r}=\mathbf{0}$ ).

More in general, one can consider multiple simultaneous human-robot contacts, e.g., the two human hands pushing on two different links. In this case, the estimation equation (10) is modified as

$$
\left(\begin{array}{c}
\widehat{\boldsymbol{F}}_{1} \\
\widehat{\boldsymbol{F}}_{2}
\end{array}\right)=\left(\begin{array}{ll}
\boldsymbol{J}_{1}^{T}(\boldsymbol{q}) & \left.\boldsymbol{J}_{2}^{T}(\boldsymbol{q})\right)^{\#} \boldsymbol{r},
\end{array}\right.
$$

where $\boldsymbol{J}_{k}$ is here the contact Jacobian for the $k$-th contact situation. As before, forces $\boldsymbol{F}_{k} \in \mathcal{N}\left(\boldsymbol{J}_{k}^{T}(\boldsymbol{q})\right)$ will not be recovered in $\widehat{\boldsymbol{F}}_{k}$, for $k=1,2$.

\section{Contact Point Detection}

In order to implement the force estimate (10), we still need to identify the position $\boldsymbol{p}_{c}$ of the contact point, which is in general unknown a priori, and then compute the associated contact Jacobian. In the common industrial HRI practice, possible contacts may only occur at the level of robot endeffector (e.g., touching a handle or the tool). We relax this restricting assumption, which would be clearly a limitation for potential applications.

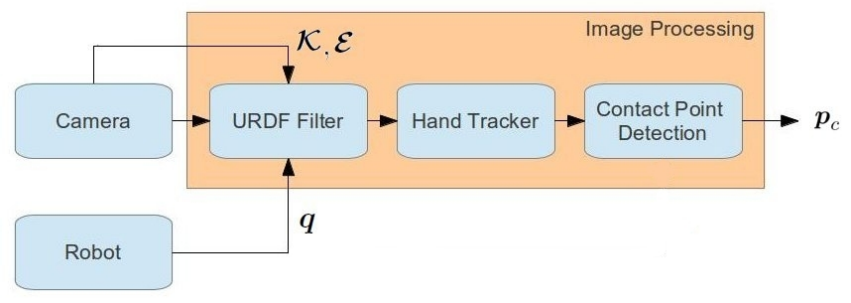

Fig. 4. Image processing scheme

To proceed in the least invasive way, we determine the contact point by using a depth image of the environment captured by a Kinect sensor, suitably placed in the environment so as to maximize visibility of the most common areas where human-robot contacts may occur [18]. With reference to Fig. 4, the depth sensor is modeled as a pin-hole camera with two sets of parameters, the intrinsic parameters in matrix $\mathcal{K}$, which models the projection of a Cartesian point on the sensor image plane, and the extrinsic parameters in matrix $\mathcal{E}$, which represents the coordinate transformation between the reference frame and the sensor frame.

A filter is used first to remove the robot from the image, starting from its URDF model [19]. Each link surface of the robot is modeled with a set of primitive shapes having a certain number of vertices. Figure 5 shows how the KUKA LWR was modeled using triangles as primitive shapes. The result is a discretization of the real surface of the robot links. The filtering operation is done by rendering the URDF robot model in the 3D depth scene, based on the joint position provided by the robot encoders, and then exploiting the rendering capability of the graphic card of the host computer. This step is needed to avoid ambiguities between the robot and human body parts (e.g., a hands) when they get very close.

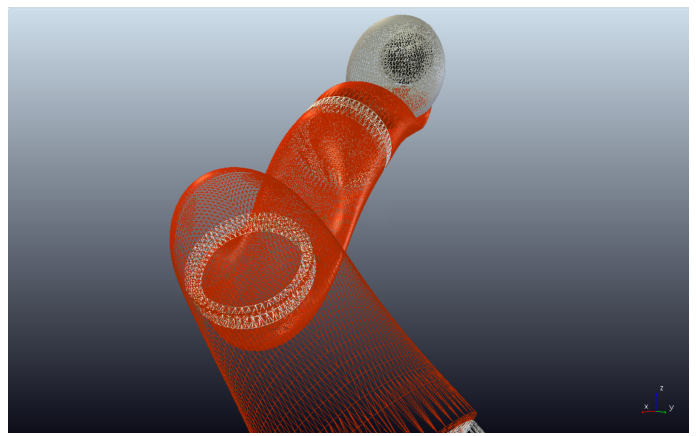

Fig. 5. The KUKA LWR robot modeled using a CAD software, with triangles as primitive shapes for the link surfaces

Most intentional physical contacts with the robot occur using our hands. The Kinect tracks the human hand [20] and provides the associated position vector $\boldsymbol{p}_{h}$. Based on this, the distance between the hand and all vertices is computed. When a contact is detected, the vertex on the link surface at minimum distance is identified as the contact point. The algorithm, presented below in pseudocode form, is initialized with the position $\boldsymbol{p}_{h}$ of the hand, as provided by the depth sensor. Note that the contact point is also updated when the hand slides along the structure of the robot. Moreover, it is easy to extend the algorithm to two hands, as well as to other body parts. In this paper, we have considered both human hands and thus up to two contact points.

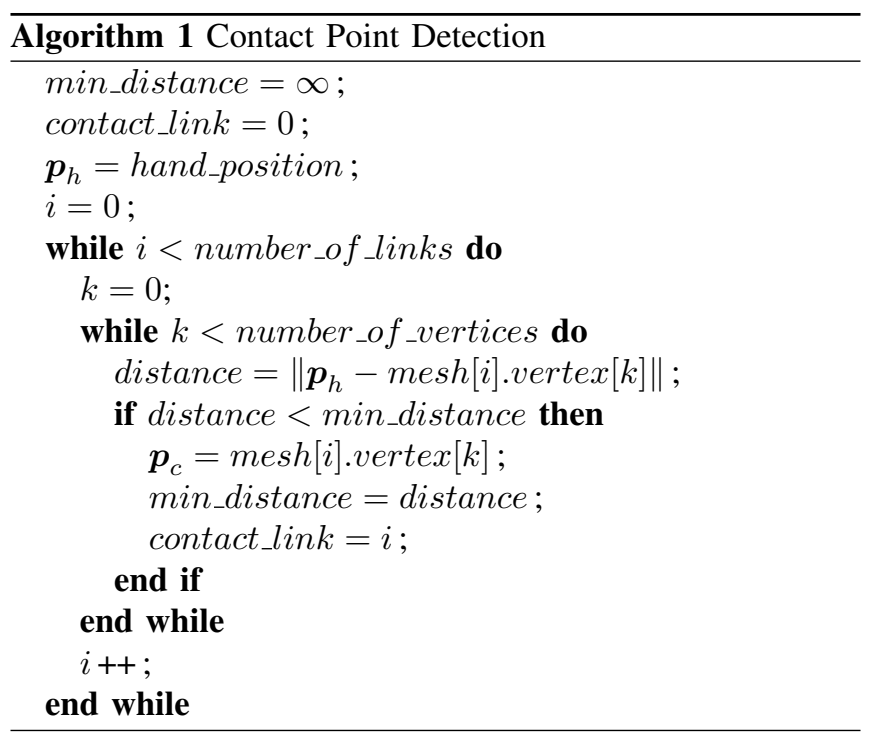

\section{EXPERIMENTAL RESULTS}

The method for estimating external contact forces and using them in a control scheme has been experimentally tested on a KUKA LWR-IV manipulator. The first step is to obtain a quantitive evaluation of the performance of 
proposed estimation process. Then, an admittance behavior at the contact point was realized by control, using the contact force estimated on line.

\section{A. Validation of contact force estimation}

In view of the dynamic nature of the problem (robot and human in motion) and the low predictability or repeatability of applied forces by humans, evaluating the accuracy of the obtained contact force estimate (including contact position) with respect to its ground truth value is not an easy task. This is especially true if no extra force/torque or tactile sensor is available. Therefore, we have performed a static validation of force estimates with the manipulator at rest, by hanging one or more known masses at different known points along the structure (see, e.g., Fig. 6). Under the action of gravity, these situations reproduce well pure contact forces applied by a human in the negative vertical direction. The validation tests have been performed with the robot in the configuration $\boldsymbol{q}=\left(\begin{array}{lllllll}0 & \pi / 2 & -\pi / 2 & -\pi / 4 & \pi / 2 & 0 & \pi / 4\end{array}\right)$.

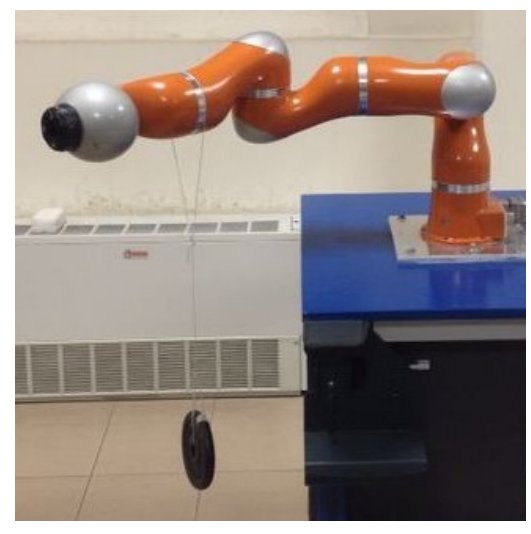

Fig. 6. A known mass hanging at a known point of link 4 of the KUKA LWR mimics the point-wise application of a pure force in static conditions, and is used for validating the proposed contact force estimation method

In the first test, a mass $m_{1}=1.93 \mathrm{~kg}$ was added (in a quasi-static way) to link 4 at the absolute position $\boldsymbol{p}_{c}=\left(\begin{array}{lll}-0.5414 & -0.1414 & 0.326\end{array}\right) \mathrm{m}$. Figure 7 shows the components of the residual vector $\boldsymbol{r}$, the Cartesian components of the force $\widehat{\boldsymbol{F}}_{c}$ estimated using the reduced $(3 \times 7)$ Jacobian $\boldsymbol{J}_{L, c}$ or those estimated using the complete $(6 \times 7)$ geometric Jacobian $\boldsymbol{J}_{c}$, and the estimated torque $\widehat{\boldsymbol{M}}_{c}$ for the latter case. Note that the last three columns of matrices $\boldsymbol{J}_{L, c}$ and $\boldsymbol{J}_{c}$ are identically zero in this situation. The component $\widehat{F}_{z}=-18.75 \mathrm{~N}$ estimated using the reduced Jacobian is very close to its true value $F_{z}=m_{1} g=18.93 \mathrm{~N}$, while the other two estimated components are practically zero as they should. When attempting to estimate $\boldsymbol{\Gamma}_{c} \in \mathbb{R}^{6}$ with the complete Jacobian, the number of informative residual components is too low $(4<6)$, and this results in a wrong estimation of both $\widehat{\boldsymbol{F}}_{c}$ and $\widehat{\boldsymbol{M}}_{c}$ (the latter should be zero while it is not, as shown in the last plot of Fig 7).

In the second test, the same mass $m_{1}$ was added to link 7 at $\boldsymbol{p}_{c}=\left(\begin{array}{lll}-0.7371 & -0.3371 & 0.326\end{array}\right) \mathrm{m}$. Figure 8 shows the relevant quantities organized as in Fig. 7. In this case,

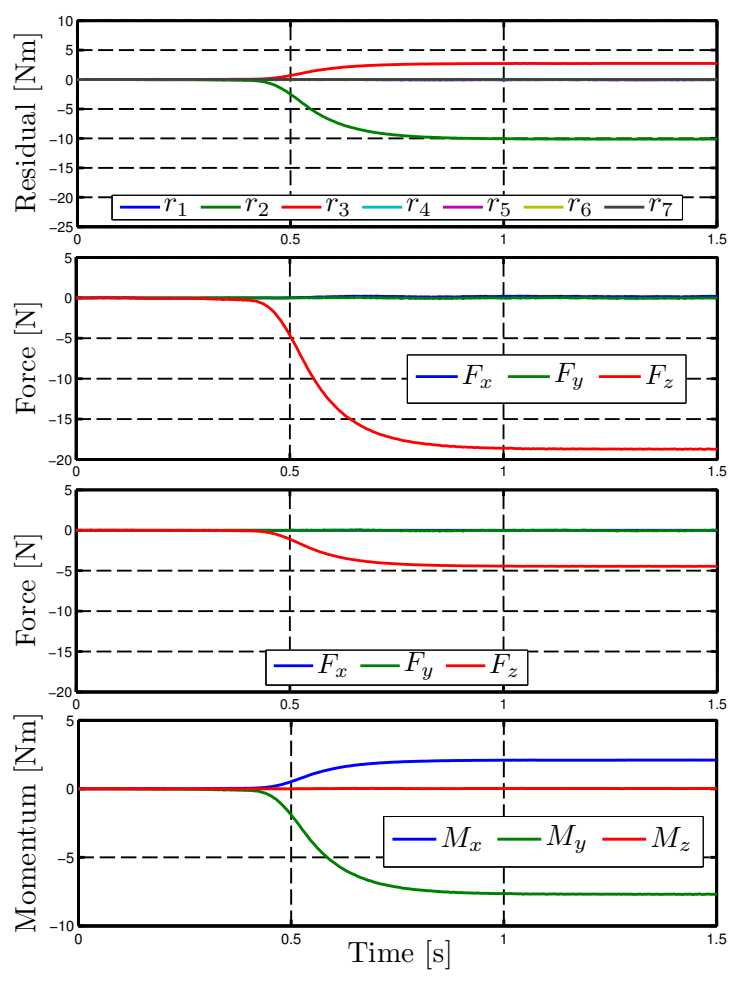

Fig. 7. First validation test with a mass on link 4: components of residual vector $\boldsymbol{r}$ (first plots at the top), $\widehat{\boldsymbol{F}}_{c}$ estimated using $\boldsymbol{J}_{L, c}$ (second), $\widehat{\boldsymbol{F}}_{c}$ and $\widehat{\boldsymbol{M}}_{c}$ estimated using $\boldsymbol{J}_{c}$ (last two at the bottom)

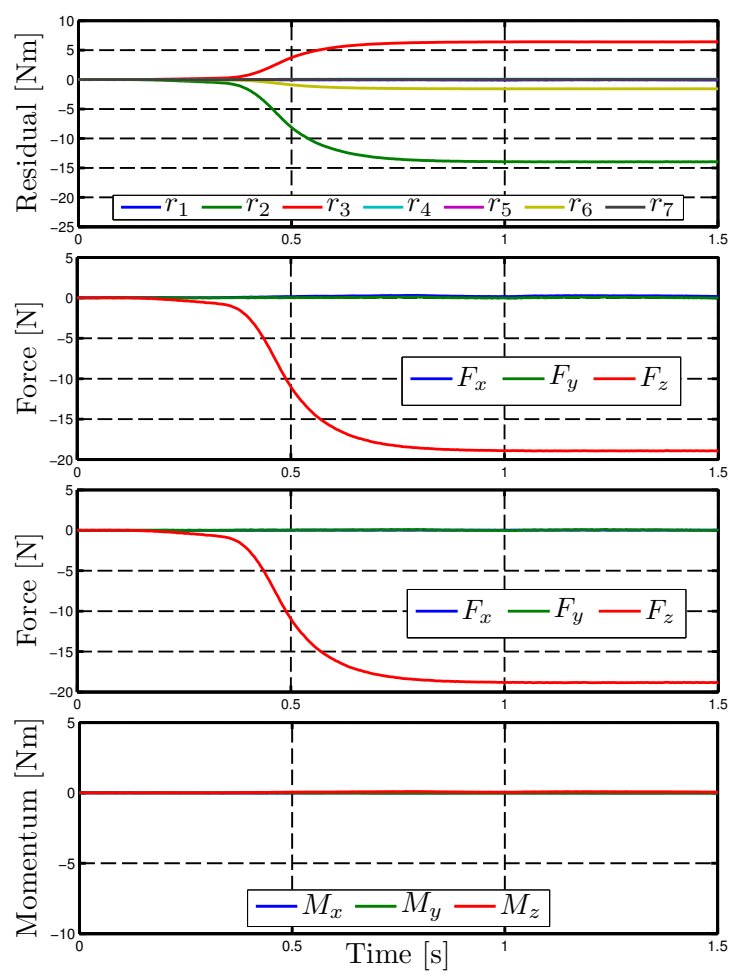

Fig. 8. Second validation test with a mass on link 7: components of residual vector $\boldsymbol{r}$ (first plots at the top), $\widehat{\boldsymbol{F}}_{c}$ estimated using $\boldsymbol{J}_{L, c}$ (second), $\widehat{\boldsymbol{F}}_{c}$ and $\widehat{\boldsymbol{M}}_{c}$ estimated using $\boldsymbol{J}_{c}$ (last two at the bottom) 


\begin{tabular}{|c|c|c|c|c|c|c|}
\hline \multicolumn{4}{|c|}{} & \multicolumn{2}{c|}{ using $\boldsymbol{J}_{L, c}$} & \multicolumn{2}{c|}{ using $\boldsymbol{J}_{c}$} \\
\hline link \# & mass $[\mathrm{kg}]$ & $F_{z}[\mathrm{~N}]$ & $\widehat{F}_{z}$ & dev & $\widehat{F}_{z}$ & dev \\
\hline 4 & 1.93 & -18.93 & -18.75 & $0.95 \%$ & -4.46 & $76.43 \%$ \\
\hline 7 & 1.93 & -18.93 & -18.91 & $0.1 \%$ & -18.82 & $0.58 \%$ \\
\hline
\end{tabular}

TABLE I

TRUE AND ESTIMATED FORCES FOR SINGLE CONTACTS

since the number of residuals $(n=7)$ is larger than the dimension of $\boldsymbol{\Gamma}_{c}(m=6)$, the estimated values of $\widehat{\boldsymbol{F}}_{c}$ and $\widehat{\boldsymbol{M}}_{c}$ are very close to the true ones with both Jacobians. The main numerical results are given in Tab. I.

A third test has been performed using two masses placed on two different links, so as to emulate a double contact situation. The first mass $m_{1}=1.93 \mathrm{~kg}$ is on link 7 at position $\boldsymbol{p}_{1}=\left(\begin{array}{lll}-0.7371 & -0.3371 & 0.326\end{array}\right) \mathrm{m}$, while the second mass $m_{2}=2.03 \mathrm{~kg}$ is on link 4 at position $\boldsymbol{p}_{2}=\left(\begin{array}{lll}-0.5414 & -0.1414 & 0.326\end{array}\right) \mathrm{m}$. The second mass has been added after a few seconds (at $t=4.9 \mathrm{~s}$ ). Figure 9 shows the residual components and the associated contact forces $\widehat{\boldsymbol{F}}_{1}$ and $\widehat{\boldsymbol{F}}_{2}$ estimated using (11). Table II compares the estimated values $\widehat{F}_{z 1}$ and $\widehat{F}_{z 2}$ with the true ones.

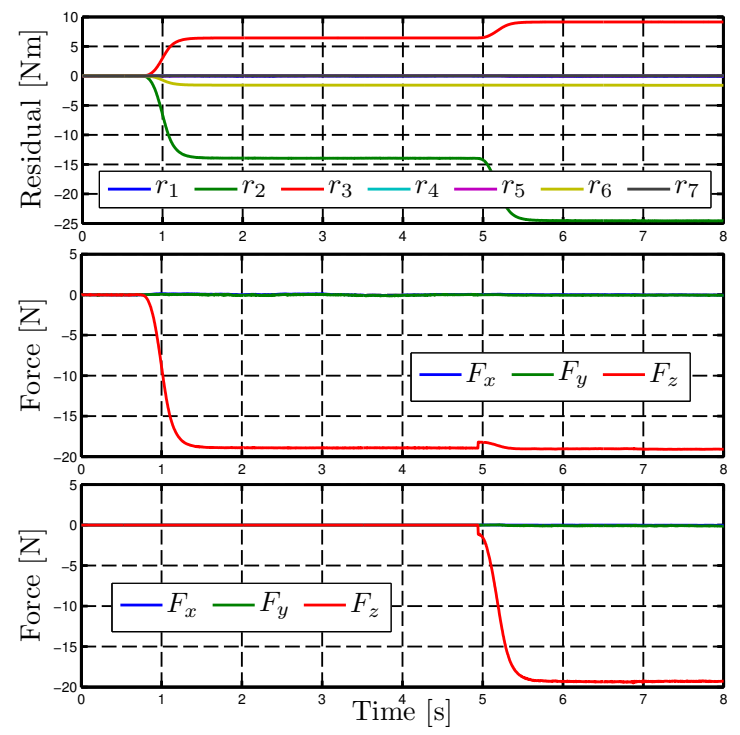

Fig. 9. Third validation test with two different masses on links 7 and 4 : components of residual vector $\boldsymbol{r}$ (top plots) and estimated components of force $\widehat{\boldsymbol{F}}_{1}$ (center) and $\widehat{\boldsymbol{F}}_{2}$ (bottom)

\begin{tabular}{|c|c|c|c|c|}
\hline link \# & mass $[\mathrm{kg}]$ & $F_{z}[\mathrm{~N}]$ & $\widehat{F}_{z}$ & $\mathrm{dev}$ \\
\hline 4 & 2.03 & -19.91 & -19.43 & $2.41 \%$ \\
\hline 7 & 1.93 & -18.93 & -19.04 & $0.58 \%$ \\
\hline
\end{tabular}

TABLE II

TRUE AND ESTIMATED FORCES FOR SIMULTANEOUS DOUBLE CONTACT ON TWO DIFFERENT LINKS

As mentioned in Sect. III-B, the interaction of a human hand with the robot can be approximated as a point contact, at least for the present purposes. To verify the sensitivity of this assumption, we pushed the robot on link 6 at the contact point $\boldsymbol{p}_{c}=\left(\begin{array}{lll}-0.376 & -0.037 & 0.668\end{array}\right) \mathrm{m}$, and considered then 10 dummy points belonging to the robot surface, placed around the actual contact point at distances less than $10 \mathrm{~cm}$ and thus mimicking a whole-hand contact. Their average position was $\boldsymbol{p}_{c, a v g}=\left(\begin{array}{lll}-0.407 & -0.071 & 0.673\end{array}\right) \mathrm{m}$, and the contact force was estimated using either $\boldsymbol{p}_{c}$ or $\boldsymbol{p}_{c, a v g}$. The comparative results in Tab. III show that the error obtained was less than $5 \%$ for the largest force component.

\begin{tabular}{|c|c|c|c|}
\hline component & $\widehat{\boldsymbol{F}}_{c}$ & $\widehat{\boldsymbol{F}}_{c, a v g}$ & error \\
\hline $\mathrm{X}$ & 2.638 & 2.613 & $0.95 \%$ \\
\hline $\mathrm{y}$ & 82.621 & 78.46 & $5.03 \%$ \\
\hline $\mathrm{z}$ & 10.37 & 10.16 & $2.02 \%$ \\
\hline
\end{tabular}

TABLE III

SENSITIVITY OF THE ESTIMATED FORCE USING A SET OF DUMMY POINTS DISTRIBUTED AROUND THE ACTUAL CONTACT POINT

\section{B. Robot control using the estimated contact force}

We have used the estimated contact forces to design various control strategies for human-robot collaboration. Here, we report on admittance control in the presence of one or two contacts. We assume that the KUKA LWR robot is in position control mode, so that the user can specify the joint velocity $\dot{\boldsymbol{q}}$ as command.

For a single contact situation, let $\boldsymbol{q}_{d}=\boldsymbol{q}\left(t_{c}\right) \in \mathbb{R}^{7}$ and $\boldsymbol{p}_{d}=\boldsymbol{p}_{c}\left(t_{c}\right) \in \mathbb{R}^{3}$ be respectively the initial robot configuration and the initial position of the contact point when the interaction with the human begins, namely at $t=t_{c}$. The position $\boldsymbol{p}_{c}(t)$ of this contact point may change for $t>t_{c}$, because the robot moves under the action of the contact force and of the chosen control law. In admittance control, the desired velocity $\dot{\boldsymbol{p}}_{c}$ of the contact point is assigned to be proportional to the (real and/or virtual) force $\boldsymbol{F}_{a}$ acting on that point, or

$$
\dot{\boldsymbol{p}}_{c}=\boldsymbol{K}_{a} \boldsymbol{F}_{a}, \quad \boldsymbol{K}_{a}=k_{a} \boldsymbol{I}>0,
$$

with (scalar) admittance gain $k_{a}$. The total active force on the contact point $\boldsymbol{p}_{c}$ is specified as

$$
\boldsymbol{F}_{a}=\widehat{\boldsymbol{F}}_{c}+\boldsymbol{K}_{p}\left(\boldsymbol{p}_{d}-\boldsymbol{p}_{c}\right), \quad \boldsymbol{K}_{p}=k_{p} \boldsymbol{I}>0,
$$

namely as the sum of the estimated contact force $\widehat{\boldsymbol{F}}_{c}$ (exerted by the human) and of a virtual elastic force, with (scalar) stiffness $k_{p}$. In this way, an equilibrium may arise $\left(\boldsymbol{F}_{a}=\mathbf{0}\right)$ between the human pushing continuously on the robot and the virtual spring pulling the contact point back to its initial position $\boldsymbol{p}_{d}$.

Since we are considering positional motion tasks only, the robot will be redundant for contact force tasks that occur on link $i \geq 4$, so that an extra null-space motion contribution can be considered. Thus, the joint velocity command to the robot will be defined in general as

$$
\dot{\boldsymbol{q}}=\boldsymbol{J}_{c}^{\#}(\boldsymbol{q}) \dot{\boldsymbol{p}}_{c}+\left(\boldsymbol{I}-\boldsymbol{J}_{c}^{\#}(\boldsymbol{q}) \boldsymbol{J}_{c}(\boldsymbol{q})\right) \dot{\boldsymbol{q}}_{0}
$$


with the additional joint velocity $\dot{\boldsymbol{q}}_{0}$ projected in the null space of the contact Jacobian $\boldsymbol{J}_{c}$. When the human breaks the contact, $\widehat{\boldsymbol{F}}_{c}$ returns rapidly to zero. In order to help the control action (13) in recovering the initial position $\boldsymbol{p}_{d}$ of the contact point, we choose as null-space joint velocity in (14)

$$
\dot{\boldsymbol{q}}_{0}=\boldsymbol{K}_{0}\left(\boldsymbol{q}_{d}-\boldsymbol{q}\right), \quad \boldsymbol{K}_{0}=k_{0} \boldsymbol{I}>0
$$

which drives the robot back to its initial configuration $\boldsymbol{q}_{d}$.

When a second contact occurs, we may treat either or both of the two contacts with a similar admittance control strategy, relating the desired velocity $\dot{\boldsymbol{p}}_{k}$ of each contact point to the associated estimate $\widehat{\boldsymbol{F}}_{k}$ of the contact force as

$$
\dot{\boldsymbol{p}}_{k}=\boldsymbol{K}_{k} \widehat{\boldsymbol{F}}_{k}, \quad \boldsymbol{K}_{k}=k_{k} \boldsymbol{I}>0, \quad \text { for } k=1,2 .
$$

However, the resulting joint velocity commands

$$
\dot{\boldsymbol{q}}=\boldsymbol{J}_{1}^{\#}(\boldsymbol{q}) \dot{\boldsymbol{p}}_{1} \quad \text { or } \quad \dot{\boldsymbol{q}}=\boldsymbol{J}_{2}^{\#}(\boldsymbol{q}) \dot{\boldsymbol{p}}_{2},
$$

where $\boldsymbol{J}_{k}$ is the Jacobian associated to the $k$-th contact, will typically result in an unsatisfactory robot behavior, or even in a conflicting situation. Therefore, we adopt a task priority strategy and consider the contribution of the contact that is located closer to the robot base (and thus more difficult to be correctly handled) as a secondary task: its associated control command will be projected in the null space of the primary contact Jacobian. Using for the higher priority contact the same control strategy as in the single contact situation, the final joint velocity will be computed as

$$
\begin{aligned}
& \dot{\boldsymbol{q}}=\boldsymbol{J}_{1}^{\#}(\boldsymbol{q}) \boldsymbol{K}_{1}\left(\widehat{\boldsymbol{F}}_{1}+\boldsymbol{K}_{p}\left(\boldsymbol{p}_{1 d}-\boldsymbol{p}_{1}\right)\right) \\
& +\left(\boldsymbol{I}-\boldsymbol{J}_{1}^{\#}(\boldsymbol{q}) \boldsymbol{J}_{1}(\boldsymbol{q})\right)\left(\boldsymbol{J}_{2}^{\#}(\boldsymbol{q}) \boldsymbol{K}_{2} \widehat{\boldsymbol{F}}_{2}+\boldsymbol{K}_{0}\left(\boldsymbol{q}_{1 d}-\boldsymbol{q}\right)\right),
\end{aligned}
$$

with obvious meaning of symbols.

Dynamic interaction experiments have been performed with the control laws (12-15) or (16) running at $5 \mathrm{~ms}$ cycle time. The workspace is monitored by a Microsoft Kinect depth sensor, positioned at a distance of $2.8 \mathrm{~m}$ behind the robot and at a height of $1.6 \mathrm{~m}$ w.r.t. the robot base frame. The Kinect captures $640 \times 480$ depth images at $30 \mathrm{~Hz}$ rate. The whole algorithm is executed on an quad-core CPU.

In the first control experiment ${ }^{2}$, the human pushes the robot on link 3 as shown in Fig. 10. The control gains are chosen so as to assign a relatively rigid behavior to the robot: $k_{a}=0.01, k_{p}=350$, and $k_{0}=0.6$. Figure 11 reports the time evolution of the residual vector, of the contact force estimate, and of the Cartesian position error of the contact point with respect to its initial position. After detecting the initial contact, the robot moves the contact point along the direction of the estimated force. Contact is maintained for about two seconds, and during this interval the human force and the robot reaction force balance each other. When the hand is removed, the contact point returns smoothly to its initial position.

In the second control experiment, the robot is pushed by the left hand on link 6, and then also by the right hand on link

\footnotetext{
${ }^{2}$ The control experiments can be viewed in the accompanying video clip.
}

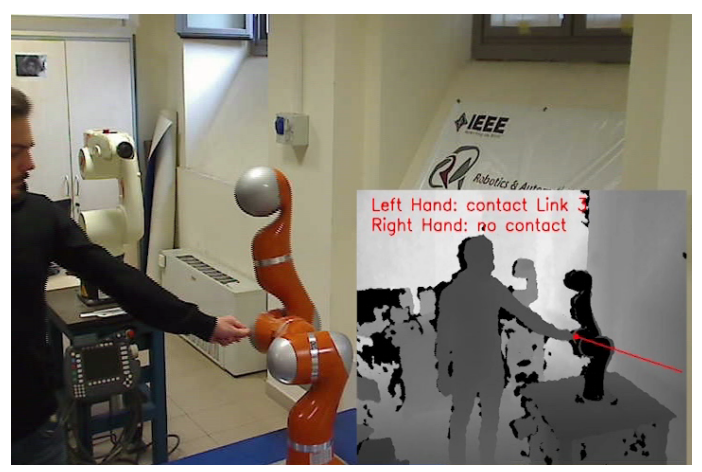

Fig. 10. Single contact between the left human hand and the robot link 3; the estimated contact force vector is highlighted in red in the depth image

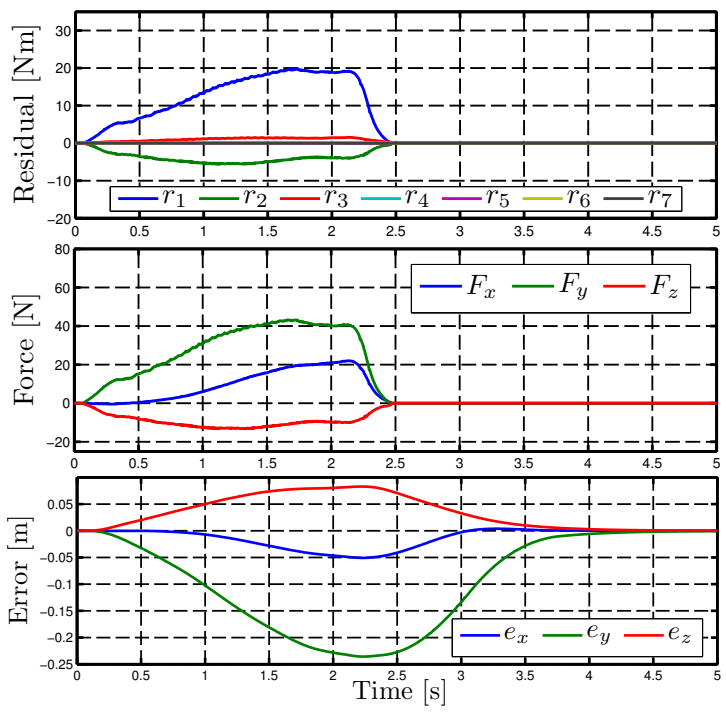

Fig. 11. First control experiment with a contact on link 3, starting at $t_{c}=0.1$ and ending at $t_{e}=2.46$; residual vector components (top), estimated contact force components (center), and Cartesian position error components (bottom)

3, as shown in Fig. 12. Figure 13 shows the residual vector, the two estimated forces $\widehat{\boldsymbol{F}}_{1}$ and $\widehat{\boldsymbol{F}}_{2}$, and the Cartesian error components when using the control law (16) with the same previous gains and the additional $k_{2}=0.03$. The overall behavior is very smooth, and the initial contact point returns to the original position when the interaction with the human is over. Moreover, when applying or removing the second contact force, the position of the first contact point remains largely unaffected, thanks to the null space projection in (16).

\section{CONCLUSION}

We have presented a new and complete approach for detecting, estimating, and handling dynamic force interactions that may occur at any point along the robot structure, in order to achieve an effective physical human-robot collaboration. The online estimation method of the contact force builds upon the momentum-based residual generator for collision detection and isolation, combined with the localization of the contact point using a depth sensor. The geometric knowledge of the contact point and the observation of the joint torques 


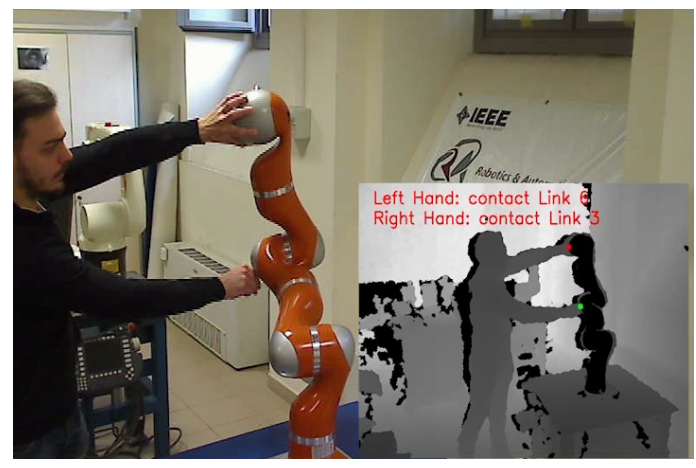

Fig. 12. Double contact between the human hands and the robot links 3 and 6 ; the estimated contact points are highlighted in red (left hand) and green (right hand) in the depth image
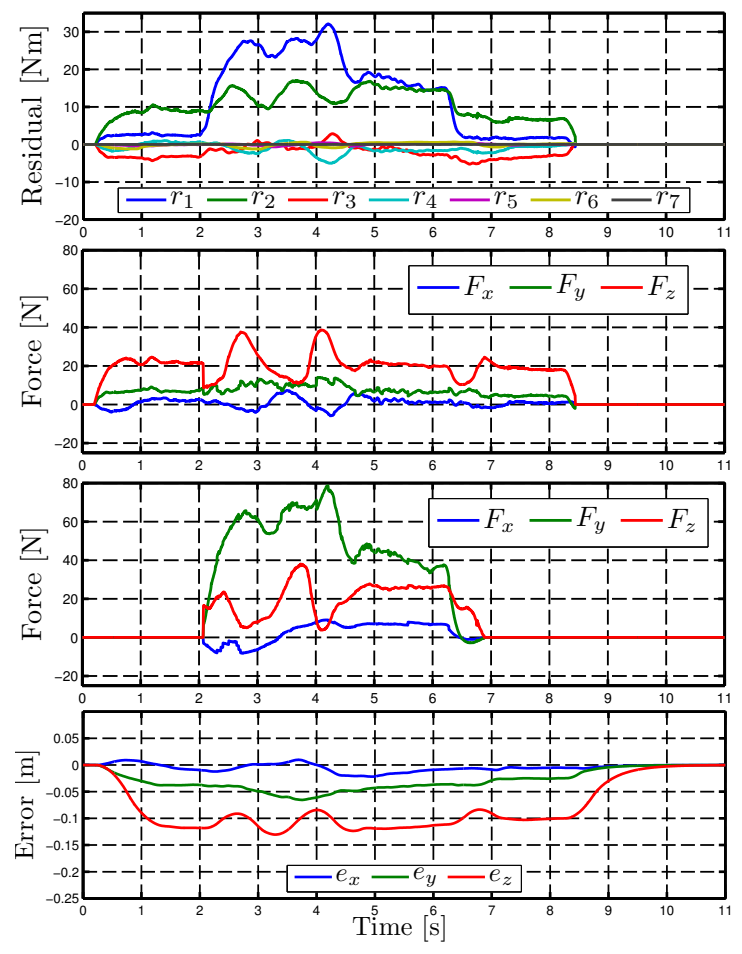

Fig. 13. Second control experiment with contact of left hand on link 6 starting at $t_{c 1}=0.2$ and of right hand on link 3 starting at $t_{c 2}=2$; (from top to bottom) residual vector $\boldsymbol{r}$, estimated force $\widehat{\boldsymbol{F}}_{1}$ due to first contact, estimated force $\widehat{\boldsymbol{F}}_{2}$ due to second contact, and Cartesian position error components

that result from the interaction are in general sufficient for obtaining a good estimate of Cartesian contact forces acting on the robot surface (e.g., those that can be applied by a human), both in single and in double contact situations.

A quantitative validation of the accuracy of the proposed estimation method was made with a number of static experiments on the KUKA LWR robot. Satisfactory performance has been obtained when feeding the contact force estimates in an admittance control law. For double contacts, the use of a simple task priority strategy was found to be a viable solution. The obtained result will serve as a basis for obtaining more complex human-robot dynamic collaborative behaviors.

\section{REFERENCES}

[1] A. De Luca and F. Flacco, "Integrated control for pHRI: Collision avoidance, detection, reaction and collaboration," in Proc. IEEE Int. Conf. on Biomedical Robotics and Biomechatronics, 2012, pp. 288295.

[2] S. Morinaga and K. Kosuge, "Collision detection system for manipulator based on adaptive impedance control law," in Proc. IEEE Int. Conf. on Robotics and Automation, 2003, pp. 1080-1085.

[3] D. Ebert and D. Henrich, "Safe human-robot-cooperation: Imagebased collision detection for industrial robots," in Proc. IEEE/RSJ Int. Conf. on Intelligent Robots and Systems, 2002, pp. 239-244.

[4] A. De Luca and R. Mattone, "Sensorless robot collision detection and hybrid force/motion control," in Proc. IEEE Int. Conf. on Robotics and Automation, 2005, pp. 1011-1016.

[5] A. De Luca, A. Albu-Schäffer, S. Haddadin, and G. Hirzinger, "Collision detection and safe reaction with the DLR-III lightweight robot arm," in Proc. IEEE/RSJ Int. Conf. on Intelligent Robots and Systems, 2006, pp. $1623-1630$.

[6] M. Geravand, F. Flacco, and A. De Luca, "Human-robot physical interaction and collaboration using an industrial robot with a closed control architecture," in Proc. IEEE Int. Conf. on Robotics and Automation, 2013, pp. 3985-3992.

[7] S. Pirozzi, A. Cirillo, and P. Cirillo, "A modular and low-cost artificial skin for robotic applications," in Proc. IEEE Int. Conf. on Biomedical Robotics and Biomechatronics, 2012, pp. 961-966.

[8] S. Kuhn and D. Henrich, "Fast vision-based minimum distance determination between known and unknown objects," in Proc. IEEE/RSJ Int. Conf. on Intelligent Robots and Systems, 2007, pp. 2186-2191.

[9] A. Petrovskaya, J. Park, and O. Khatib, "Probabilistic estimation of whole body contacts for multi-contact robot control," in Proc. IEEE Int. Conf. on Robotics and Automation, 2007, pp. 568-573.

[10] F. Alonge, A. Bruno, and F. D'Ippolito, "Interaction control of robotic manipulators without force measurement," in Proc. IEEE Int. Symp. on Industrial Electronics, 2010, pp. 3245-3250.

[11] N. Hogan, "Impedance control: An approach to manipulation: Part I - Theory, Part II - Implementation, Part III - Applications," ASME J. of Dynamic Systems, Measurement, and Control, vol. 107, pp. 1-24, 1985.

[12] L. Zollo, B. Siciliano, A. De Luca, E. Guglielmelli, and P. Dario, "Compliance control for an anthropomorphic robot with elastic joints: Theory and experiments," ASME J. of Dynamic Systems, Measurement, and Control, no. 127, pp. 321-328, 2005.

[13] A. Albu-Schäffer, C. Ott, and G. Hirzinger, "A unified passivitybased control framework for position, torque and impedance control of flexible joint robots," Int. J. of Robotics Research, vol. 26, pp. 23-39, 2007.

[14] C. Ott, Cartesian Impedance Control of Redundant and Flexible-Joint Robots, ser. Springer Tracts in Advanced Robotics (STAR). Springer, 2008.

[15] H. Sadeghian, M. Keshmiri, L. Villani, and B. Siciliano, "Null-space impedance control with disturbance observer," in Proc. IEEE/RSJ Int. Conf. on Intelligent Robots and Systems, 2012, pp. 2795-2800.

[16] D. E. Whitney, "Force feedback control of manipulator fine motions," ASME J. of Dynamic Systems, Measurement, and Control, vol. 99, no. 2, pp. 91-97, 1977.

[17] S. Haddadin, A. Albu-Schäffer, A. De Luca, and G. Hirzinger, "Collision detection and reaction: A contribution to safe physical humanrobot interaction," in Proc. IEEE/RSJ Int. Conf. on Intelligent Robots and Systems, 2008, pp. 3356-3363.

[18] F. Flacco and A. De Luca, "Multiple depth/presence sensors: Integration and optimal placement for human/robot coexistence," in Proc. IEEE Int. Conf. on Robotics and Automation, 2010, pp. 3916-3923.

[19] Realtime URDF filter. [Online]. Available: http://github.com/blodow/ realtime_urdf_filter

[20] PrimeSense. [Online]. Available: http://www.primesense.com 